\title{
Posttraumatische Dissektion der Arteria renalis - bildgebende Diagnostik und interventionelle Therapie
}

Polytraumatisierte Patienten zeigen im Bereich der parenchymatösen intra- und retroperitonealen Organe bei entsprechender Gewalteinwirkung Leber-, Milzund Nierenrupturen. Gefäßabrisse mit entsprechenden Blutungen führen meist zu unmittelbaren operativen Konsequenzen. Posttraumatische Dissektionen der Nierenarterien sind insgesamt selten. Sie sind bei fehlendem Hämatom in der primären Bildgebung schwierig zu erfassen und drohen im weiteren Verlauf zu einem Verschluss der Nierenarterie zu führen.

Vorgestellt wird der Fall einer posttraumatischen Dissektion der linken Nierenarterie mit entsprechender Diagnostik und erfolgreicher interventionell-radiologischer Therapie in Form einer Stentimplantation.

Veranschaulicht werden soll dabei besonders die Strategie der bildgebenden Diagnostik in Form einer primären kontrastmittelunterstützten Spiral-CT-Untersuchung und einer befundbezogenen farbkodierten Duplexsonographie. Die
Die linke Arteria und Vena renalis waren nur minimal kontrastiert und eine kortikomedulläre Differenzierung des Parenchyms nur noch angedeutet erkennbar. Es bestand lediglich ein kleines pararenales Hämatom (Abb.1). Unter dem Verdacht einer Dissektion wurde eine farbkodierte Duplexsonographie durchgeführt. Diese zeigte eine minimale Restdurchblutung in der Peripherie der Niere mit abgeflachten systolischen peaks und einem Widerstandsindex von $\mathrm{RI}=0,35$ im Sinne der systolischen Einstrombehinderung. Im mittleren Drittel der linken Nierenarterie fanden sich massive Turbulenzen mit perivaskulären Farbartefakten und Flussbeschleunigungen auf über $5 \mathrm{~m} / \mathrm{s}$ (Abb. 2).

Es schloss sich die intraarterielle digitale Subtraktionsangiographie an. Die selektive Darstellung der linken Niere zeigte eine ausgeprägte Dissektion im mittleren Drittel der Arteria renalis mit geringer Restdurchblutung des Nierenparenchyms (Abb.3a). Die extrarenale Aufzweigung der A. renalis täuscht eine Fortsetzung der Dissektion nach weiter distal reichend vor, als in Wirklichkeit vorhanden, was mit zusätzlichen Projektionen bewiesen wurde. Mittels V-18Draht (Meditech, Boston Scientific Corporation ${ }^{\circledR}$, Watertown, USA) erfolgte die vorsichtige Sondierung der Dissektionsstelle und nachfolgend die Implantation eines $5 / 14 \mathrm{~mm}$ ballonmontierten Stents (Bridge Stent ${ }^{\circledR}$ AVE) mit vollständiger Wiederherstellung des normalen Lumens der Arterie (Abb. $\mathbf{3}$ b). Der Stent endet distal vor der extrarenalen Aufzweigung der Nierenarterie. Die nachfolgenden Kontrollen in der Duplexsonographie und im CT zeigten eine vollständige Normalisierung der Nieren-

einem Verkehrsunfall kreislaufstabil und intubiert mit Prellmarken an Thorax und Abdomen eingeliefert. Die orientierende Sonographie zeigte keine größere Menge freier Flüssigkeit intraabdominell oder retroperitoneal bei Verdacht auf eine Milzkontusion. Die nachfolgende Spiral-CT-Untersuchung (Tomoscan AV, Fa. Philips Medizin Systeme ${ }^{\circledR}$ ) wurde unter Gabe von $120 \mathrm{ml}$ Accupaque 300 (Nycomed $^{\circledR}$ ) mit einer Flussrate von $2,5 \mathrm{ml} / \mathrm{s}$ und einem Delay von $30 \mathrm{~s} \mathrm{kra-}$ niokaudal von der oberen Thoraxapertur bis zur Symphyse durchgeführt. Bei einer Kollimation von $7 \mathrm{~mm}$ und einem Tischvorschub von $7 \mathrm{~mm} / \mathrm{s}$ (pitch $1: 1$ ) betrug der Rekonstruktionsindex ebenfalls $7 \mathrm{~mm}$. Dabei zeigte sich neben schweren Lungen- und Milzkontusionen die linke Niere ausgeprägt minderdurchblutet.
Abb.1 Spiral-CT des Abdomens (7/7/7) mit KM i.v.: Minderdurchblutung der linken Niere mit Restenhancement. Die Nierenarterie zeigt noch eine partielle Kontrastierung.

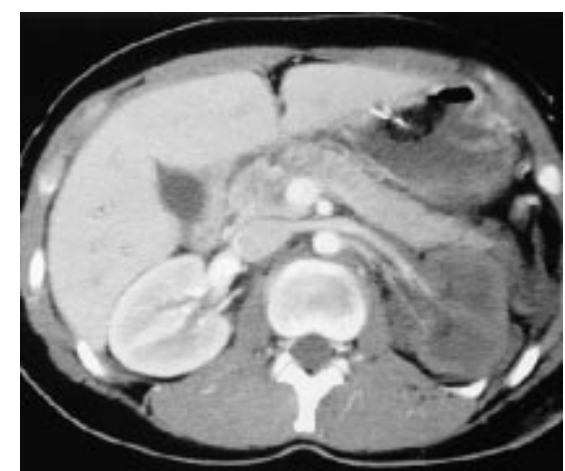




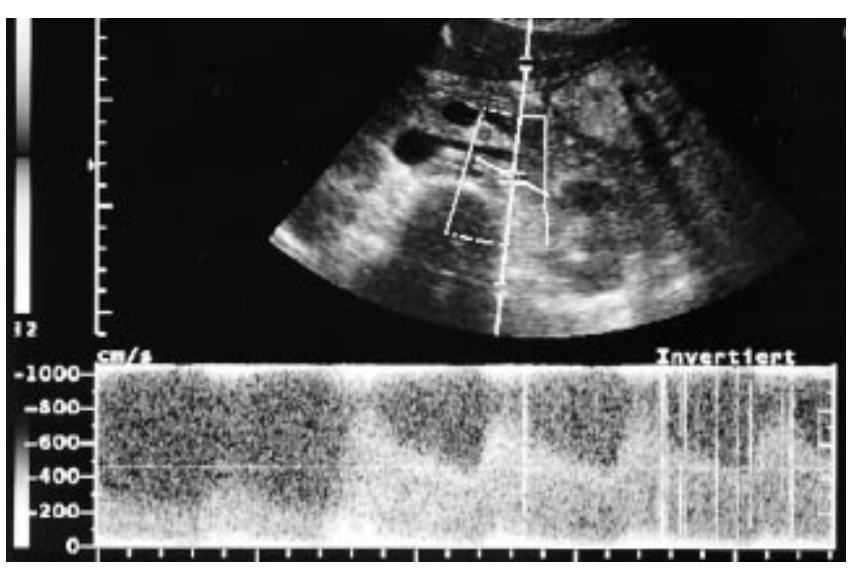

durchblutung mit regelrechten Nierenwerten.

\section{Diskussion}

Verletzungen der Nieren als Traumafolge stellen sich bildgebend meist als Rupturen, Kontusionen oder Hämatome dar. Der Gefäßstiel, wenn er nicht von einem Abriss betroffen ist, lässt sich im Rahmen der Akutdiagnostik wesentlich schwieriger abklären. Gerade jedoch die Dissektion mit erhaltener Restdurchblutung des Parenchyms erfordert eine schnelle und effektive Diagnostik und Therapie, um den drohenden vollständigen Gefäßverschluss zu verhindern.

Literaturangaben zufolge (Welling RE et al., Ann Vasc Surg 1989; 3: 345) führen insbesondere bei polytraumatisierten Patienten Dissektionen der Nierenarterien zum Organverlust mittels Nephrektomie, da die Diagnose nach mehr als 24 Stunden gestellt wird und die Thrombose dann irreversibel ist. Andere Leitverletzungen stehen häufig im Vordergrund und lenken Diagnostik und Therapie vom Problem der Nierendurchblutung $\mathrm{ab}$.

Es findet sich in der Literatur bisher kein vergleichbarer Fall der Dissektion einer Nierenarterie bei einem jungen Patienten ohne arteriosklerotische Veränderungen nach einem Polytrauma, der nach zeitgerechter Diagnostik erfolgreich mit einer Stentimplantation therapiert werden konnte.
Abb. 2 Die Duplexsonographie der linken Nierenarterie zeigt eine extreme Geschwindigkeitserhöhung, die sich erst im high-PRF-Modus darstellen lässt $\left(14 \mathrm{KHz}, \mathrm{v}_{\max } 8 \mathrm{~m} / \mathrm{s}\right)$

Die bisher beschriebenen Dissektionen beziehen sich v.a. auf postinterventionelle Verletzungen der Nierenarterien nach perkutanen Ballondilatationen (Mahler Fet al., Nephron 1986: 44: Suppl 1: 60-63). Ein anderer Mechanismus besteht in Dissektionen der Aorta, die die Nierenarterien sekundär mit einbeziehen (Gates JD et al., J Trauma 1994, 36: 430).

Posttraumatisch bedingte Dissektionen der Nierenarterien finden sich in der Literatur nur in wenigen Fällen, die dann chirurgisch behandelt wurden (Slavis SA et al., J Urol. 1990: 144: 233). Gewertz et al. (Arch Surg 1977; 112: 409) fanden als wichtigste Symptome Hypertension, Makrohämaturie und Schmerz. Daraus wurde auch die Indikation zur Angiographie abgeleitet. Diese Vorgehensweise ist bei polytraumatisierten Patienten nicht praktikabel, weshalb wir das primäre Spiral-CT mit intravenöser Kontrastmittelgabe zur Objektivierung des Befundes favorisieren. Wesentlich erscheint dabei die Kontrastmittelapplikation im richtigen Zeitintervall. Die Kontrastierung von Nierenarterie und Nierenvene sowie eine ausreichende kortikomedulläre Differenzierung im Parenchym zur genauen Beurteilung der Durchblutung sind unerlässlich.

Die weitere Differenzierung der Pathologie an der Nierenarterie mit Hilfe farbkodierter Duplexsonographie stellt ohne wesentlichen Zeitverlust vor dem Einsatz eines invasiven Verfahrens eine
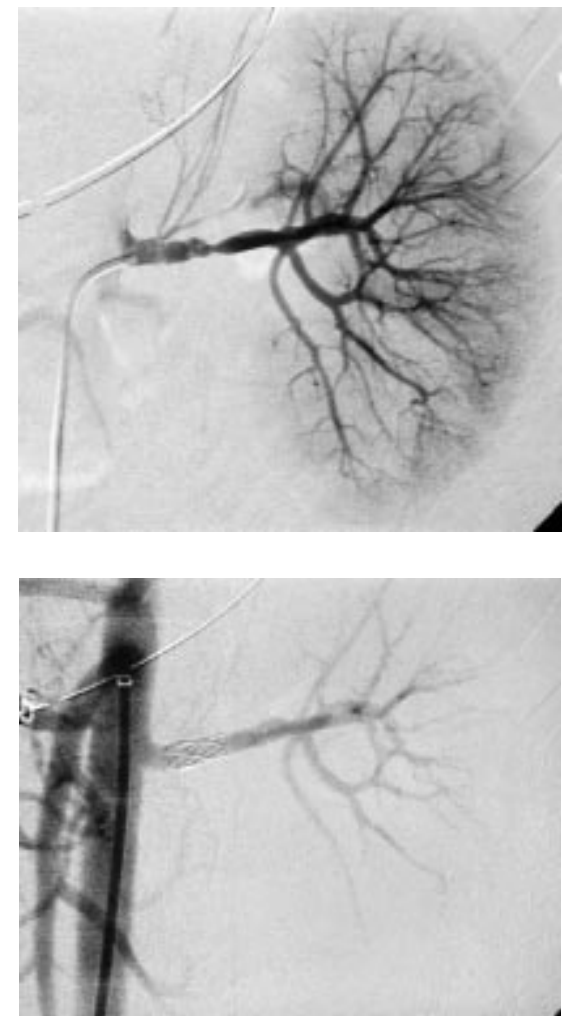

Abb. 3 (a) In der selektiven Renovasographie hochgradige Stenosierung mit Dissektionszeichen. Zeitige Aufzweigung der Nierenarterie extrarenal. (b) Mittels Stent versorgte linke Nierenarterie mit Wiederherstellung der regelrechten Durchblutung. Der Stent endet vor der extrarenalen Aufzweigung der Arterie.

sinnvolle Methodenergänzung dar. Bei erfahrenem Untersucher und bei guter Einsehbarkeit gelingt die Diagnosestellung zuverlässig und die Therapieentscheidung kann getroffen werden.

Die interventionell-radiologische Therapie mittels Stentimplantation ist einer offen chirurgischen vorzuziehen, da bei weiteren Verletzungen - wie in unserem Fall die Milz- und Lungenkontusionen eine Operation den Gesamtzustand des Patienten verschlechtern könnte.

S. Mutze, St. Paris, R. Laun, Berlin 